\title{
Markov Process of Muscle Motors
}

\author{
Yu. Kondratiev ${ }^{1}$, E. Pechersky ${ }^{2}$, S. Pirogov ${ }^{2}$ \\ ${ }^{1}$ Bielefeld University, 33601 Bielefeld, Germany \\ 2 IITP, 19, Bolshoj Karetny per., GSP-4, Moscow 127994, Russia \\ kondrat@mathematik.Uni-Bielefeld.DE, pech@iitp.ru, pirogov@iitp.ru
}

\begin{abstract}
We study a Markov random process describing a muscle molecular motor behavior. Every motor is either bound up with a thin filament or unbound. In the bound state the motor creates a force proportional to its displacement from the neutral position. In both states the motor spend an exponential time depending on the state. The thin filament moves at its velocity proportional to average of all displacements of all motors.

We assume that the time which a motor stays at the bound state does not depend on its displacement. Then one can find an exact solution of a non-linear equation appearing in the limit of infinite number of the motors.
\end{abstract}




\section{Introduction}

Recent progress in the molecular motors study brought a wave of new models and methods in theoretical considerations. The tools involved into considerations are spread from biochemistry and biophysics to mathematics and probability theory. As examples, we would like to mention works [1, 2, 3, 8, 6]. This list contains only some papers from different areas.

We concentrate here on mathematical aspects of the problem and, more precisely, we describe a probabilistic model of the muscle motor which leads to a non-linear Markov process. The latter notion was introduced by E.P.McKean [7]. Comparing with the usual case, in a non-linear Markov process transition probabilities are related to a non-linear equation. With respect to the probability theory, non-linear Markov processes play main role in the modelling. Because of non-linearity, the model appears to have many effects atypical for usual Markov processes that gives possibilities either to explain experimentally observed properties of the motors or to predict new ones. Following Howard' book [10] we shall distinguish two classes of the molecular motors: processive motors and non-processive ones. The distinction between these classes leads to different types of the models, however differences are relative. Perhaps one can construct a general model yielding all features of both sorts of motors. We consider here a model of non-processive motors which concerns motors involved in the muscle activity.

\section{Model}

\subsection{Informal description}

Main components of a molecular motor complex are protein molecules called the motors themselves which perform a motion along a molecule called filament (or microtubule) and playing a role of railway for the motors. At last, there exists a cargo or back bond which play passive role of a relocatable ingredient. As it can be extracted from the biochemical and biophysical literature, there are several kinds of the motor protein molecules involved in slightly different types of movements.

In this work we study the motors producing muscle motions. Those motors 
are fixed by one of their end at a long protein molecule called the thick filament. The thick filament is fastened in a cell. Thus the motors do not move with respect to the cell they are located in. The second end of the motors may be attached or not attached to a thin filament, another long molecule in the cell disposed parallel to the thick filament. If both ends of a motor are attached then the motor can cause a tension acting on the thin filament if the end positions are not opposite each other. This tension is the cause of the thin filament motion. The binding to and unbinding out the thin filament of any motor are random. When binding a motor chooses a point on the thin filament to attach randomly. The tension the motor creates is defined by a displacement value between two ends of the motor. Therefore all bounded motors create different tensions. It is common to take the velocity of the thin filament moving proportional to the average of all bounded filament tensions.

\subsection{Formal description}

The formalization of the muscle motor construction informally described above can be done in the following way. We introduce a random process of interacted particles. Every particle represent a motor. Therefore we locate the particles at points of $\mathbb{Z}$.

Let us start with a random process $\zeta_{k}(t)$ describing the binding and unbinding process the particle located at $k \in \mathbb{Z}$. The state space is the two-point set $D=\{0,1\}$, where 0 means the unbinding and 1 means the binding particle state. Then the infinitesimal operator of $\zeta_{k}(t)$ is the following $2 \times 2$ matrix

$$
L_{k}^{D}=\left(\begin{array}{cc}
-c_{b} & c_{b} \\
c_{u} & -c_{u}
\end{array}\right)
$$

where $\operatorname{Pr}\left(\zeta_{k}(t)=1 / \zeta_{k}(0)=0\right)=c_{b} t+o(t)$ and $\operatorname{Pr}\left(\zeta_{k}(t)=0 / \zeta_{k}(0)=1\right)=$ $c_{u} t+o(t)$.

Then the probability $P_{k}(t)$ of $k \mathrm{~h}$ motor to be unbound at the time $t$ satisfies the equation

$$
\frac{\mathrm{d} P_{k}(t)}{\mathrm{d} t}=-c_{b} P_{k}(t)+c_{u}\left(1-P_{k}(t)\right)
$$

which gives in the steady state

$$
P_{k}=\frac{c_{u}}{c_{b}+c_{u}} .
$$


The state space $\mathbb{X}$ of $k \mathrm{~h}$ motor consists of pairs $(z, \varepsilon)$ where $z \in \mathbb{R}$ if $\varepsilon=1$ and $z=0$ if $\varepsilon=0$. As before the parameter $\varepsilon$ indicates the bounded (if $\varepsilon=1$ ) and unbounded (if $\varepsilon=0$ ) positions of the particle. We define a random process $\xi_{k}(t)$ describing binding and unbinding actions and the displacement when binding, including the deterministic moving of a single particle. Let $b(x)$ be a distribution density such that $\int x b(x) \mathrm{d} x>0$. The infinitesimal operator of the process defining the behaviour of $k$ th particle is

$$
\begin{array}{r}
L_{k} f(x, \varepsilon)=c_{b}\left[\int b(z) f(z, 1-\varepsilon) \mathrm{d} z-f(x, \varepsilon)\right](1-\varepsilon)- \\
\varkappa x \frac{\mathrm{d}}{\mathrm{d} x} f(x, \varepsilon) \varepsilon+c_{u}[f(k, 1-\varepsilon)-f(0, \varepsilon)] \varepsilon,
\end{array}
$$

Now a constant $c_{b}>0$ is the rate of the particle to jump to a point of $\mathbb{R} \times\{1\}$ from the state $(0,0)$, that is $\operatorname{Pr}\left(\zeta_{k}(t) \in \mathbb{R} \times\{1\} / \zeta_{k}=(0,0)\right)=c_{b} t+o(t)$. A constant $c_{u}>0$ is the rate of the particle to jump to $(0,0)$ from any point in $\mathbb{R} \times\{1\}$. That means that the rate of unbinding does not depend on the point $x \in \mathbb{R}$ where the particle was attached at the unbinding moment. The function $b(x)$ is the probability density of the particle to bind at the point $x$ if the particle was at $(0,0)$. Here $x$ is the displacement of the particle with respect to its neutral position. Constant $\varkappa$ is positive. It is the tension of the single motor molecule. It can be seen from (5) that when the particle is on $\mathbb{R}$, that is $\varepsilon=1$, then it is moving to the point 0 with the velocity proportional to $x$.

For the density $p_{k}(x, t)$ of the particle to be on $\mathbb{R}$ we obtain the following differential equation (see [12])

$$
\frac{\partial p_{k}(x, t)}{\partial t}=c_{b} b(x) P_{k}(t)+\varkappa \frac{\partial}{\partial x}\left[x p_{k}(x, t)\right]-c_{u} p_{k}(x, t),
$$

where $P_{k}(t)=1-\int p_{k}(x, t) \mathrm{d} x$. The probability $P_{k}(t)$ to be unbound satisfies (1).

Next we introduce the interaction between the particles. We cannot express the interaction in a Hamiltonian form. Instead we introduce a deterministic dynamic of all bound particles such that particles dynamic is highly correlated each to other. Moreover all particles are moving with the same velocity. To be more precise consider all particles in the interval $[-N, N] \subset \mathbb{Z}$ and the configuration space $\Omega_{N}=\mathbb{X}^{[-N, N]}$ of all particles in $[-N, N]$. The space $\Omega_{N}$ 
is a disjoint union of the sets $\Omega_{\varepsilon_{-N}, \ldots \varepsilon_{N}}=\prod_{i} \mathbb{R}^{\varepsilon_{i}}$. We can consider any probability distribution on $\Omega_{N}$ as a collection of measures on spaces $\Omega_{\varepsilon_{-N}, \ldots \varepsilon_{N}}$.

The generator of the process involving all particles from $[-N, N]$ is

$$
\begin{aligned}
& L_{[-N, N]} f\left(\left(x_{k}, \varepsilon_{k}\right), k=-N, \ldots, N\right)= \\
& c_{b} \sum_{k=-N}^{N}\left[\int b(z) f\left(\ldots,\left(x_{k-1}, \varepsilon_{k-1}\right),\left(z, 1-\varepsilon_{k}\right),\left(x_{k+1}, \varepsilon_{k+1}\right), \ldots\right) \mathrm{d} z-\right. \\
& \left.f\left(\left(x_{k}, \varepsilon_{k}\right), k=-N, \ldots, N\right)\right]\left(1-\varepsilon_{k}\right)- \\
& +c_{u} \sum_{k=-N}^{N}\left[f\left(\ldots,\left(x_{k-1}, \varepsilon_{k-1}\right),\left(k, 1-\varepsilon_{k}\right)\left(x_{k+1}, \varepsilon_{k+1}\right), \ldots\right)\right. \\
& \left.-f\left(\left(x_{k}, \varepsilon_{k}\right), k=-N, \ldots, N\right)\right] \varepsilon_{k}- \\
& v_{N} \sum_{k=-N}^{N} \frac{\partial}{\partial x_{k}} f\left(\left(x_{k}, \varepsilon_{k}\right), k=-N, \ldots, N\right) \varepsilon_{k},
\end{aligned}
$$

where $v_{N}=\varkappa \frac{1}{2 N+1} \sum_{k=-N}^{N} x_{k} \varepsilon_{k}-F$. The term $F$ in above formula means the velocity which an external force adds to the common velocity $\widehat{v}_{N}=$ $\frac{1}{2 N+1} \sum_{k=-N}^{N} x_{k} \varepsilon_{k}$ of all particles.

Further, we use the following notations. Let $\left(\varepsilon_{-N}, \ldots, \varepsilon_{N}\right)$ be fixed. Then $M^{0}=\left\{i:-N \leq i \leq N, \varepsilon_{i}=0\right\}$ and $M^{1}=\left(M^{0}\right)^{c}=\left\{i:-N \leq i \leq N, \varepsilon_{i}=\right.$ $1\}$.

From now we shall denote the vector $\left(\left(x_{k}, \varepsilon_{k}\right), k=-N, \ldots, N\right)$ by $X$.

For a configuration $X=\left(\left(x_{k}, \varepsilon_{k}\right), k=-N, \ldots, N\right)$ and $i \in M^{1}$ define the configuration $u_{i} X$ for which the pair $\left(x_{i}, 1\right)$ is substituted by $(0,0)$; for $i \in M^{0}$ and $x \in \mathbb{R}$ define $b_{i}^{x} X$ as the configuration for which $\left(0, \varepsilon_{i}=0\right)$ is substituted by $(x, 1)$.

Let $p^{N}(X, t)$ be the measure density of all bound particles to be at the given 
points at the time moment $t$. Then $p^{N}(X, t)$ satisfies the equation

$$
\begin{aligned}
& \frac{\partial p^{N}(X, t)}{\partial t}+\sum_{i=-N}^{N} \frac{\partial}{\partial x_{i}}\left[\varepsilon_{i} v_{N} p^{N}(X, t)\right]= \\
& c_{b} \sum_{i=-N}^{N} \varepsilon_{i} b\left(x_{i}\right) p^{N}\left(u_{i} X, t\right)+c_{u} \sum_{i=-N}^{N}\left(1-\varepsilon_{i}\right) \int p^{N}\left(b_{i}^{x} X, t\right) \mathrm{d} x- \\
& {\left[c_{u} \sum_{i=-N}^{N} \varepsilon_{i}+c_{b} \sum_{i=-N}^{N}\left(1-\varepsilon_{i}\right)\right] p^{N}(X, t) .}
\end{aligned}
$$

Let $p_{k}^{N}(x, t)$ be the probability density of the $k$-th particle to be at the point $x$ at the time moment $t$.

We are interested in the behavior of the motor system for the large number of motors which formally corresponds to the limit $N \rightarrow \infty$. In this limit we can substitute $v_{N}$ by its expectation value

$$
v=\frac{\varkappa}{2 N+1} \sum_{i=-N}^{N} \int x p_{k}^{N}(x, t) \mathrm{d} x-F
$$

Plugging in $v$ into (6) we get the nonlinear equation corresponding to nonlinear Markov process describing interacting motors.

Let us denote by

$$
\nu_{X}(\mathrm{~d} x)=\frac{1}{2 N+1} \sum_{i=-N}^{N} \varepsilon_{i} \delta_{x_{i}}(\mathrm{~d} x)
$$

the random measure describing the motor distribution on the thin filament. The expectation of this random measure is called the first correlation measure and its density is called the first correlation function, $n(x, t)$. It is evident that

$$
n(x, t)=\frac{1}{2 N+1} \sum_{k=-N}^{N} p_{k}^{N}(x, t) .
$$

For the non-linear Markov process defined above the first correlation function $n(x, t)$ satisfies the equation

$$
\frac{\partial n(x, t)}{\partial t}+v \frac{\partial n(x, t)}{\partial x}=c_{b} b(x)(1-N(t))-c_{u} n(x, t)
$$


where $N(t)=\int n(x, t) \mathrm{d} x$ and $v$ as above is

$$
v=-\varkappa \int x n(x, t) \mathrm{d} x+F
$$

It follows from (9) that $N(t)$ and $v(t)$ satisfy the equations

$$
\begin{aligned}
& \dot{N}=c_{b}(1-N)-c_{u} N \\
& \dot{v}=-\varkappa\left(v N+c_{b}(1-N) m_{1}\right)+c_{u}(F-v),
\end{aligned}
$$

where $m_{1}=\int x b(x) \mathrm{d} x$. Evidently for $t \rightarrow \infty N$ and $v$ tend to their limit values

$$
\begin{aligned}
& \bar{N}=\frac{c_{b}}{c_{b}+c_{u}}, \\
& \bar{v}=-\frac{\varkappa \bar{N} m_{1}-F}{1+\varkappa \frac{N}{c_{u}}}=-\frac{\varkappa c_{b} m_{1}-F\left(c_{b}+c_{u}\right)}{c_{b}+c_{u}+\varkappa \frac{c_{b}}{c_{u}}}
\end{aligned}
$$

Let us study the dependence of $\bar{v}$ on $c_{u}$ and $c_{b}$. It is clear that $\bar{v} \rightarrow 0$ as $c_{u} \rightarrow 0$, and $\bar{v} \rightarrow F$ as $c_{u} \rightarrow \infty$. If $\varkappa m_{1}-F>0$ then $\frac{\mathrm{d} \bar{v}}{\mathrm{~d} c_{u}}<0$ at $c_{u}=0$ and hence there exists a value $c_{u}^{o}$ where $\bar{v}$ is negative and maximal in absolute value $|\bar{v}|=v^{o}$. It is achieved at

$$
c_{u}^{o}=\sqrt{\frac{F^{2}}{m_{1}^{2}}+c_{b} \frac{\varkappa m_{1}-F}{m_{1}}}-\frac{F}{m_{1}}
$$

If there is no the external force $F=0$ then $c_{u}^{o}=\sqrt{c_{b} \varkappa}$ and

$$
v^{o}=\frac{\sqrt{c_{b}} m_{1}}{2 \sqrt{\varkappa}+\sqrt{c_{b}}} .
$$

In the case $F>0$ the velocity becomes positive for large the unbound intensity $c_{u}>\frac{c_{b}\left(\varkappa m_{1}-F\right)}{F}$.

Recall that all above values was obtained under the condition of small external force $F<\varkappa m_{1}$. If $F>\varkappa m_{1}$ then the velocity is positive at any $c_{u}$. 
Let us consider more general case when the returning force depends on $x$ non-linearly, so instead of (10) we have

$$
v=\int \varphi(x) n(x, t) \mathrm{d} x+F,
$$

where $\varphi(x)$ is some non-linear function.

Now we substitute the expression (15) in the equation (91). The expression for the time derivative of $v$ is

$$
\dot{v}=\int \varphi \dot{n} \mathrm{~d} x=-v \int \varphi \frac{\partial n}{\partial x} \mathrm{~d} x+c_{b}(1-N(t)) \int \varphi b(x) \mathrm{d} x-c_{u}(v-F) .
$$

Integrating by parts we obtain from the first term $v \int \varphi^{\prime}(x) n(x, t) \mathrm{d} x$. Inserting the new variable $w=\int \varphi^{\prime}(x) n(x, t) \mathrm{d} x$ we have $\dot{w}=\int \varphi^{\prime}(x) \dot{n}(x, t) \mathrm{d} x$ and using (9) again we get an expression for $\dot{w}$ containing the integral $\int \varphi^{\prime \prime}(x) n(x, t) \mathrm{d} x$.

If $\varphi(x)$ is a polynomial then repeating this procedure we finally obtain a finite system of ordinary differential equations.

The similar substitution is valid if $\varphi(x)$ is any trigonometric polynomial or more generally any linear combination of quasi-polynomials (i.e. usual polynomials multiplied by sinusoidal and exponential functions [13]). Consider the simplest case $\varphi(x)=-\varkappa \sin (\alpha x)$. Denoting $w=\int \cos (\alpha x) n(x, t) \mathrm{d} x$ and $m_{c}=\int \cos (\alpha x) b(x) \mathrm{d} x, m_{s}=\int \sin (\alpha x) b(x) \mathrm{d} x$ we have

$$
\begin{aligned}
\dot{N} & =c_{b}(1-N)-c_{u} N \\
\dot{v} & =-\varkappa \alpha v w-\varkappa c_{b}(1-N) m_{s}+c_{u}(F-v) \\
\dot{w} & =\frac{\alpha v(v-F)}{\varkappa}+c_{b}(1-N) m_{c}-c_{u} w .
\end{aligned}
$$

For any stationary point of this system the value $N$ is given by (12) as before, and $v$ and $w$ can be found from $\dot{v}=\dot{w}=0$. It is interesting to find the number of the stationary points of (17). Also it is interesting to study the limit cycles for this system if they exist.

For the case $\varphi(x)=-\varkappa \sinh (\alpha x)$ the equation for $N, v, w$ are similar to (17) with the only difference in the sign before the term $\frac{\alpha v(v-F)}{\varkappa}$. 


\section{Acknowledgements}

The authors thank P. Reimann for valuable discussions on molecular motors. Financial support of the SFB-701, Bielefeld University, is gratefully acknowledged. E.P. and S.P. were partially supported by the CRDF Grant RUM1-2693-MO-05.

\section{References}

[1] Juelicher F and Prost J, Spontaneous Oscillations of Collective Molecular Motors 1997 Phys. Rev. Lett. 784510

[2] Parmeggiani A, Juelicher F, Peliti L and Prost J, Detachment of molecular motors under tangential loading 2001 Europhys. Lett. 56603

[3] Jeulicher F and Prost J, Cooperative Molecular Motors 1995 Phys. Rev. Lett. 752618

[4] Jeulicher F, Ajdari A and Prost J, Modeling molecular motors 1997 Reviews of Modern Physics, 691269

[5] Juelicher F and Prost J, Molecular Motors: From Individual to Collective Behavior, 1998 Progress of Theoretical Physics Supplement 1309

[6] Maes C and van Wieren M, Model for Kinesin 2003 Journal of Statistical Physics 112329

[7] Mc-Kean, H.P., A class of Markov processes associated with non-linear parabolic equations 1966 Proc. NAS 561907

[8] Reimann P, Brownian motors: noisy transport far from equilibrium 2002 Physics Reports 36157

[9] James Keener and James Sneyd, Mathematical Physiology, Springer, 1998

[10] J. Howard, Mechanics of Motor Proteins and the Cytoskeleton, Sinauer Associates, 2001 
[11] H.C. Taylor, M.E.J. Holwill, Axonemal dynein - a natural molecular motor, Sixth conference on molecular nanotechnology

[12] I.J Gihman, A.V. Skorohod, Stochasic Differential Equations, Springer Verlag, 1972.

[13] L.S. Pontryagin, Ordinary Differential Equations, R\&C Dynamics, 2001 